\title{
Pentacyclic Triterpenes from Terminalia arjuna Show Multiple Benefits on Aged and Dry Skin
}

\author{
M. Farwick ${ }^{a} \quad$ T. Köhler ${ }^{a} \quad$ J.Schild ${ }^{a} \quad$ M. Mentel $^{a} \quad$ U. Maczkiewitz ${ }^{a} \quad$ V. Paganic \\ A. Bonfiglic L. Riganoc ${ }^{c}$ D. Bureik ${ }^{b} \quad$ G.G. Gauglitz ${ }^{b}$ \\ ${ }^{a}$ Evonik Industries AG, Essen, and b Department of Dermatology and Allergology, Ludwig Maximilians University, \\ Munich, Germany; ' Institute of Skin and Product Evaluation (ISPE), Milan, Italy
}

\section{Key Words}

Arjunolic acid · Triterpenes · Antiaging properties .

Skin blood microcirculation · Skin penetration

\begin{abstract}
Background: Pentacyclic triterpenoids improve epidermal barrier function and induce collagen production. Here, their effects on cutaneous aging by means of objective instrumental measurements were elucidated. Methods: Reconstituted human epidermis, cultivated keratinocytes and fibroblasts were incubated with Terminalia arjuna triterpenes (T. arjuna bark extract), and mRNA and protein expression of various genes was determined using microarray analysis, qRT-PCR and ELISA techniques. Clinical efficacy of T. arjuna bark extract versus vehicle control cream was elucidated in 30 patients and transepidermal water loss (TEWL), skin hydration and elasticity were measured. Another 30 female patients in their postmenopausal phase were treated with a similar regime, and skin sebum content, cutaneous blood microcirculation and skin density/echogenicity were assessed. Results: Incubation with T. arjuna triterpenes increased FGF-2, TSP-1, TGF- $\beta$ and CTGF expression, and VEGF secretion in vitro. Elevated lactate dehydrogenase release
\end{abstract}

upon sodium dodecyl sulphate challenge was reversed by the application of $T$. arjuna bark extract. T. arjuna bark extract decreased TEWL, improved skin moisturization, reduced scaliness and led to significantly improved skin elasticity. Also, increases in blood microflow and skin sebum content as well as improved skin thickness/echogenicity were noted on postmenopausal skin, resulting in visible reduction of sagging skin on the jowls as demonstrated by digital photography. Conclusion: T. arjuna bark extract appears as an innovative active ingredient that exerts versatile antiaging properties in vitro and in vivo.

ㄷ) 2013 S. Karger AG, Basel

\section{Introduction}

Skin aging represents a highly complex but not yet fully understood progressive, multifaceted process [1]. It is currently thought that a variety of different factors contribute to the global expression of skin aging. Similar to the entire organism, the skin is subject to an unpreventable intrinsic aging process. Cellular aging and shortening of telomeres, mutations of mitochondrial DNA, oxidative stress, genetic mutations and decrease of several

\section{KARGER}

E-Mail karger@karger.com

www.karger.com/spp
(C) 2013 S. Karger AG, Basel

$1660-5527 / 14 / 0272-0071 \$ 38.00 / 0$
Gerd G. Gauglitz, MD

Department of Dermatology and Allergology, Ludwig Maximilians University Frauenlobstrasse 9-11

DE-80337 Munich (Germany)

E-Mail Gerd.Gauglitz@med.uni-muenchen.de 
hormone levels are currently thought to contribute to intrinsic aging [2]. In particular, oestrogens affect several skin functions and the oestrogen deprivation that accompanies menopause contributes to, and exacerbates, the deleterious effects of age on the skin [3]. In addition, skin aging is also significantly influenced by exogenous factors [1] such as chronic UV light exposure [4]. The formation of reactive oxygen species and the induction of matrix metalloproteinases (MMPs) reflect the central aspects of both intrinsic and extrinsic cutaneous aging [1]. Despite some morphological and pathophysiological differences underlying the various types of skin aging, all of them share some common characteristics such as dermal atrophy, loss of collagen density, reduction in dermal cell populations and decreased microvasculature [5]. Other commonly experienced physiological changes observed during cutaneous aging include impairment of barrier function, xerosis, loss of elasticity and slower turnover of epidermal cells [6].

Also, a progressive reduction in water-binding capacity and changes in cutaneous permeability for chemical substances has been observed [7]. However, proper sustenance of the skin is very important, since it represents the largest organ of the human body and needs to fulfil multiple metabolic tasks.

The first evidence of clinical signs associated with skin aging is often found in the periorbital area and includes wrinkles, eyelid bags, circles around the eye, or a 'tired' look [8]. Anti-aging and moisturizing products claim to counteract the visible signs of aging, mainly by stimulating dermal processes and assisting the skin to maintain an appropriate level of humidity. However, only a few cosmetic preparations are available which have proven efficient based on objective quantitative methods.

Triterpenoids constitute the major components of many traditional medicinal plants [9]. They represent a large family of complex structures synthesized in plants via cyclisation of squalene and have been used in traditional Asian medicine for centuries [9]. They are well known for their anti-inflammatory, immunomodulatory, hepatoprotectory, analgetic and antitumour effects [911]. In addition, pentacyclic triterpenoids have been described in the literature for their various beneficial effects on skin, including improvement of epidermal barrier function, promotion of keratinocyte differentiation and induction of collagen production [12-14], and thus have potential in targeting typical features of cutaneous aging.

In this study, we aimed to elucidate the effects of a cosmetic cream containing predominantly triterpenoids as the active principle, which were obtained by extraction from the bark of the arjun tree. Skin absorption properties were determined and several in vivo and in vitro parameters commonly utilized to describe cutaneous aging were assessed by means of objective instrumental measurements.

\section{Materials and Methods}

The active ingredients in Terminalia arjuna bark extract are pentacyclic triterpenes which were obtained by extraction from the bark of the arjun tree. Briefly, T. arjuna bark is cleaned, cut and pulverized. The resulting coarse powder is extracted at elevated temperature with organic solvent. The extract is filtered and concentrated under vacuum to a paste. The product is purified by 2 additional extractions and a filtration step before it is crystallized to yield a fine powder. Dissolving about $8 \%$ of the powder in pentylene glycol yields a standardized extract consisting of approximately $90 \%$ triterpenoids of which $60-65 \%$ are arjunolic acid and $25-30 \%$ are asiatic acid. The biologically active form of T. arjuna triterpenes ( $T$. arjuna bark extract) has some obstacles when it comes to processing in cosmetic formulations at physiologically relevant concentrations. T. arjuna bark extract is neither soluble in water nor in most cosmetic oils. It can only be formulated at low concentrations in a high amount of a very hot and polar oil phase or as a solution in ethanol or pentanediol. Due to these formulation restrictions, TEGO $^{\circledR}$ Arjuna $S$ was developed. TEGO ${ }^{\circledR}$ Arjuna $\mathrm{S}$ is a standardized solution of T. arjuna bark extract in 1,2-pentanediol with a minimum of $7 \%$ triterpenoids.

In vitro Culturing of Reconstituted Human Epidermis Models

Reconstituted human epidermis was purchased from SkinEthic (Nice, France) and incubated for $24 \mathrm{~h}$ in standard maintenance medium at $37^{\circ} \mathrm{C}$ and $5 \% \mathrm{CO}_{2}$ as previously described [15] before starting the experiments.

In order to evaluate the general biological effects of T. arjuna bark extract, the reconstituted human epidermis was topically treated with a thin liquid $(\mathrm{O} / \mathrm{W})$ formulation containing $0.5 \% T$. arjuna bark extract for 12 or $24 \mathrm{~h}$, respectively. Reconstituted human epidermis treated with an $\mathrm{O} / \mathrm{W}$ formulation without active ingredient served as control. Subsequently, tissues were harvested and lysed and the total RNA was isolated.

For assessment of gene expression, total RNA was extracted after incubation as described elsewhere [7]. DNA chip technique was used to evaluate the effect of T. arjuna bark extract on the genetic level of human skin models. In brief, RNA samples were transcribed into cDNA serving as a template for the in vitro generation of fluorescently labelled RNA. Affymetrix HGU133 plus 2.0 GeneChips (Affymetrix Inc., Santa Clara, Calif., USA) were used to characterize genome-wide expression using $2 \mu \mathrm{g}$ of total RNA pooled from three reconstituted human epidermis skin models. Gene chip assays and initial analysis were carried out as previously described [16]. After screening, TaqMan analysis of specific transcripts for vascular endothelial growth factor (VEGF), fibroblast growth factor receptor 2 (FGFR2) and thrombospondin-1 (TSP-1) was performed with predesigned and optimized Assayson-Demand (Applied Biosystems). Relative quantification was performed using 18S-rRNA as reference as described earlier [17]. 
Gene and Protein Analysis on Human Epidermal

Keratinocytes

Primary normal human epidermal keratinocytes cultivated under non-differentiating conditions were treated with 5 and $10 \mathrm{ppm}$ T. arjuna bark extract, $10 \mathrm{ppm}$ Centella asiatica triterpenes or 10 ppm soy isoflavones for 6 or $24 \mathrm{~h}$, respectively; 1,2-pentanediol $(0.05 \%)$ was used as vehicle control. Samples were harvested and snap frozen until RNA isolation was performed. The cell culture supernatant was stored at $-20^{\circ} \mathrm{C}$ for further protein analysis. Gene expression of VEGF, fibroblast growth factor (FGF-2), TSP-1, transforming growth factor $\beta$ (TGF- $\beta$ ), connective tissue growth factor (CTGF) and glycerin aldehyde phosphate dehydrogenase (GAPDH) were determined using qRT-PCR as described before [7]. The following gene-specific primers were used. VEGF: forward 5'-CTACCTCCACCATGCCAAGT- $3^{\prime}$ and reverse $5^{\prime}$-GCGAGTCTGTGTTTTTGCAG-3'; FGF-2: forward $5^{\prime}$-GGAGAAGAGCGACCCTCAC- $3^{\prime}$ and reverse $5^{\prime}$-AGCCAGGTAACGGTTAGCAC$3^{\prime}$; TSP-1: forward $5^{\prime}$-GCCTGATGACAAGTTCCAAGA- $3^{\prime}$ and reverse $5^{\prime}$-CTTTGCGATGCGGAGTCT-3'; TGF- $\beta$ : forward $5^{\prime}$ GCCCTGGACACCAACTATTGCT- $3^{\prime}$ and reverse $5^{\prime}$-AGGCTCCAAATGTAGGGGCAGG-3' ${ }^{\prime}$, and CTGF: forward $5^{\prime}$-TGCCCGGGAAATGCTGCGAG-3' ${ }^{\prime}$ and reverse $5^{\prime}$-TGCACAGGCGGCTCTGCTTC-3'. GAPDH served as a reference gene with the following primer pair: forward $5^{\prime}$-ACCACAGTCCATGCCATCAC- $3^{\prime}$ and reverse $5^{\prime}$-TCCACCACCCTGTTGCTGTA- $3^{\prime}$.

Production of VEGF protein was analysed in the cell culture supernatant using common ELISA techniques (Invitrogen Corporation, Camarillo, Calif., USA) according to the manufacturer's instructions.

\section{Overall Gene Expression Analysis (GeneChips) on Human \\ Dermal Fibroblasts}

Cultured primary human dermal fibroblasts were cultivated with 5 and $10 \mathrm{ppm}$ T. arjuna bark extract, $10 \mathrm{ppm} C$. asiatica triterpenes, $10 \mathrm{ppm}$ soy isoflavones or $10 \mathrm{ng} / \mathrm{ml} \mathrm{TGF}-\beta$ (positive control) for $6 \mathrm{~h}$; 1,2-pentanediol (0.05\%) was used as vehicle control. Samples were harvested and snap frozen until RNA isolation was performed. DNA chip technique was used to evaluate global effects of $T$. arjuna bark extract on gene expression in human dermal fibroblasts.

\section{Cellular Protection against External Stress}

To elucidate the potential protecting effect of T. arjuna bark extract against external stress, reconstituted human epidermal skin models (SkinEthic) were topically treated with $0.5 \%$ T. arjuna bark extract solubilized in an appropriate vehicle (propylene glycol) for $24 \mathrm{~h}$. Application of propylene glycol served as vehicle control. Afterwards, skin models were challenged for $40 \mathrm{~min}$ with $0.25 \%$ sodium dodecyl sulphate (SDS, solution in phosphate-buffered saline, $\mathrm{PBS}, \mathrm{pH} 7.4$ ) to create cell damage and tissue irritation. Subsequently, the treatment with the test solutions (vehicle vs. $T$. arjuna bark extract) for $24 \mathrm{~h}$ was repeated. Cell culture supernatants were collected $48 \mathrm{~h}$ after the second application for analysis of lactate dehydrogenase (LDH) release as cell damage marker. Additionally, $52 \mathrm{~h}$ after SDS treatment cell viability was determined by XXT (2,3-bis-(2-methoxy-4-nitro-5-sulfophenyl)-2Htetrazolium-5-carboxanilide) photometric assay (Cell Proliferation Kit II, Roche Applied Science).

Pentacyclic Triterpenes from T. arjuna

Show Benefits on Aged and Dry Skin
Penetration Properties of T. arjuna Bark Extract in an ex vivo Pig Skin Model

The penetration properties of T. arjuna bark extract were determined in the context of a cosmetic emulsion. On the one hand, the extract was added to the oil phase of the formulation, while, on the other hand, a presolution of T. arjuna bark extract in 1,2-pentanediol (TEGO ${ }^{\circledR}$ Arjuna S) was added to the emulsion at the end of the production process. The formulations were analysed with respect to skin penetration and formulation stability.

The study is based on the OECD guideline (OECD Guideline for the Testing of Chemicals, Skin Absorption: in vitro Method, TG 428, adopted 13 April 2004). Application conditions were chosen to mimic reality as closely as possible. The integrity of the porcine skin was examined with caffeine.

Porcine skin was used to determine the cutaneous absorption of the test substance applied in formulation. For the oil phase of the emulsion PPG-3 myristyl ether (TEGOSOFT ${ }^{\circledR}$ APM) was used as relatively polar oil, caprylic/capric triglyceride (TEGOSOFT $^{\circledR}$ CT) was used as a representative of a medium polar oil and mineral oil was used as a non-polar emollient.

A skin layer of a defined thickness (about 1,000 $\mu \mathrm{m}$ ) containing the stratum corneum, epidermis and part of the dermis was prepared with a dermatome. Punched circular pieces of porcine skin were placed in suitable diffusion chambers (Franz diffusion cells) for the testing. Approximately $20 \mathrm{mg} / \mathrm{cm}^{2}$ of the test formulations were applied on the porcine skin and the dermis side of the porcine skin disc was in contact with receptor fluid during the experiment. The diffusion cells were placed in a climate chamber at defined conditions $\left(32^{\circ} \mathrm{C}, 50 \%\right.$ relative humidity) for $24 \mathrm{~h}$ reflecting in-use conditions. After $24 \mathrm{~h}$, the residual test formulation was collected from the skin surface with a cotton swab, and the skin was cut into small pieces for extraction. Finally, the amount of active ingredient from the cotton swab, the skin extraction and the receptor fluid were determined analytically.

Improvement of Skin Moisturization and Elasticity in vivo

In order to investigate the effects of T. arjuna bark extract on skin moisturization and elasticity 30 panellists (mixed panel) were recruited. The study was conducted in wintertime. A total of 15 volunteers received an $\mathrm{O} / \mathrm{W}$ formulation containing $0.25 \% \mathrm{~T}$. arjuna bark extract, while the other 15 panellists received the formulation without active ingredient (vehicle). Each panellist applied the test formulation twice daily for 8 weeks on the inner forearm. Prior to application and after 4 weeks of treatment transepidermal water loss (TEWL) and skin hydration were measured with a Tewameter TM 300 and a Corneometer CM 825, respectively (Courage + Khazaka, Cologne, Germany), as previously described [7].

In addition, skin scaliness was determined using the Visioscan VC 98 (Courage + Khazaka) prior to application of the formulation and 4 weeks after usage [7].

The effect of $T$. arjuna bark extract on skin elasticity was determined after 8 weeks by measuring $\mathrm{R} 1$ and $\mathrm{R} 5$ using a $\mathrm{Cu}$ tometer MPA 580 (Courage + Khazaka) [7]. R1 represents the ability of the skin to return to its original state $(\mathrm{R} 1=\mathrm{Uf}-\mathrm{Ua})$ [18]. R5 is considered the net elasticity of the skin without the viscous deformation. Elastic recovery is represented by the ratio of the immediate retraction to immediate distension $(\mathrm{R} 5=\mathrm{Ur} /$ Ue) [19]. The closer the value is to $1(100 \%)$, the more elastic the skin is. 


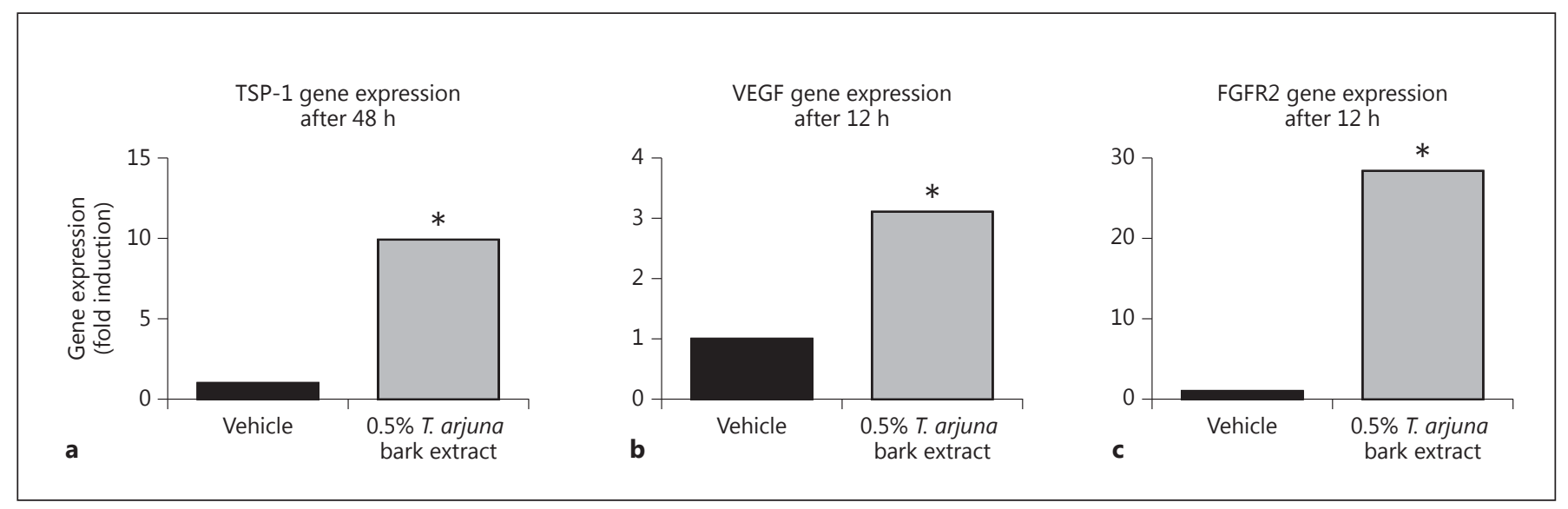

Fig. 1. TSP-1 (a), VEGF (b) and FGFR2 (c) gene expression in human reconstituted epidermis models after treatment with $T$. arjuna bark extract at different time points measured by qRT-PCR. The results are calculated relative to vehicle. ${ }^{*} \mathrm{p}<0.05$ vs. vehicle.

All measurements were carried out under standardized conditions in a climate room at $22^{\circ} \mathrm{C}$ and $50-52 \%$ relative humidity. The panellists were resting under these climate conditions for a minimum of $15 \mathrm{~min}$ before determination of skin parameters.

Reducing Symptoms of Postmenopausal Skin in vivo

In order to evaluate the effect of T. arjuna bark extract on characteristic symptoms of postmenopausal skin, including reduced sebum production, microcirculation and skin thickness, 40 Caucasian women (aged 50-70 years) in their postmenopausal period were included in a subsequent in vivo study. Exclusion criteria were any kind of skin disease and recent usage of anti-wrinkle treatments. The volunteers were instructed to avoid any additional facial creams and to circumvent exposure to UV light during the study period.

Each volunteer received two formulations, which were applied twice daily on either side of the face. T. arjuna bark extract was tested at a concentration of $0.2 \%$. The formulation was prepared by adding a solution of T. arjuna bark extract in 1,2-pentanediol to the emulsion after the homogenization step below $40^{\circ} \mathrm{C}$. The tested concentration equals $2.5 \% \mathrm{TEGO}^{\circledR}$ Arjuna S. Soy isoflavones were tested as market benchmark for mature skin care. A formulation without active ingredient was used as a negative control.

At baseline and after 4, 8, and 12 weeks of application, skin sebum content was measured using a Sebumeter SM 810 (Courage + Khazaka), according to the manufacturer's instructions. Cutaneous blood microcirculation was determined using a Flowmeter Periflux PF4001 (Perimed, Stockholm, Sweden) which is based on the laser Doppler method. With this technique the total local microcirculatory blood perfusion including the perfusion in capillaries (nutritive flow), arterioles, venules and shunting vessels can be determined. A beam of laser light emitted by a fibre-optic probe is partially reflected and partially absorbed by the examined tissue. Light hitting moving blood cells undergoes a change in wavelength (Doppler shift), while light hitting static objects is unchanged. The perfusion is expressed in perfusion units which are arbitrary units determined by the Doppler device.
Skin density/echogenicity was assessed with an ultrasound scanner, Dermascan $C^{\circledR}$ (Cortex Technology, Hadsund, Denmark) in B-scan mode and expressed in percent. Digital images were taken for clinical evaluation after receiving written consent from the patient.

\section{Statistical Analysis}

Results are presented as mean \pm SEM (in vitro $n=3$ per group at each time point, unless otherwise stated). The data were analysed using Student's t test, and differences were considered significant at a $\mathrm{p}$ value of $<0.05$, unless otherwise stated.

\section{Results}

\section{Treatment with T. arjuna Bark Extract Leads to}

Altered Gene Expression in Cutaneous Cells in vitro

Microarray analysis of reconstituted human epidermis treated with $T$. arjuna bark extract revealed a significant gene regulatory activity of this compound. A total of 42 genes were at least 2-fold up- or downregulated after 12 or $48 \mathrm{~h}$ (data not shown). For selected candidates, the results of the microarray analysis were verified by qRT-PCR using the TaqMan method (fig. 1).

Treatment of human reconstituted epidermis with $T$. arjuna bark extract resulted in approximately a 10-fold increase of TSP-1 gene expression after $48 \mathrm{~h}$ of application compared to vehicle (fig. 1a). Also, a 3-fold increase in VEGF and a 30-fold increase in FGFR2 gene expression after $12 \mathrm{~h}$ of application were noted when compared to vehicle (fig. $1 b, c)$. 


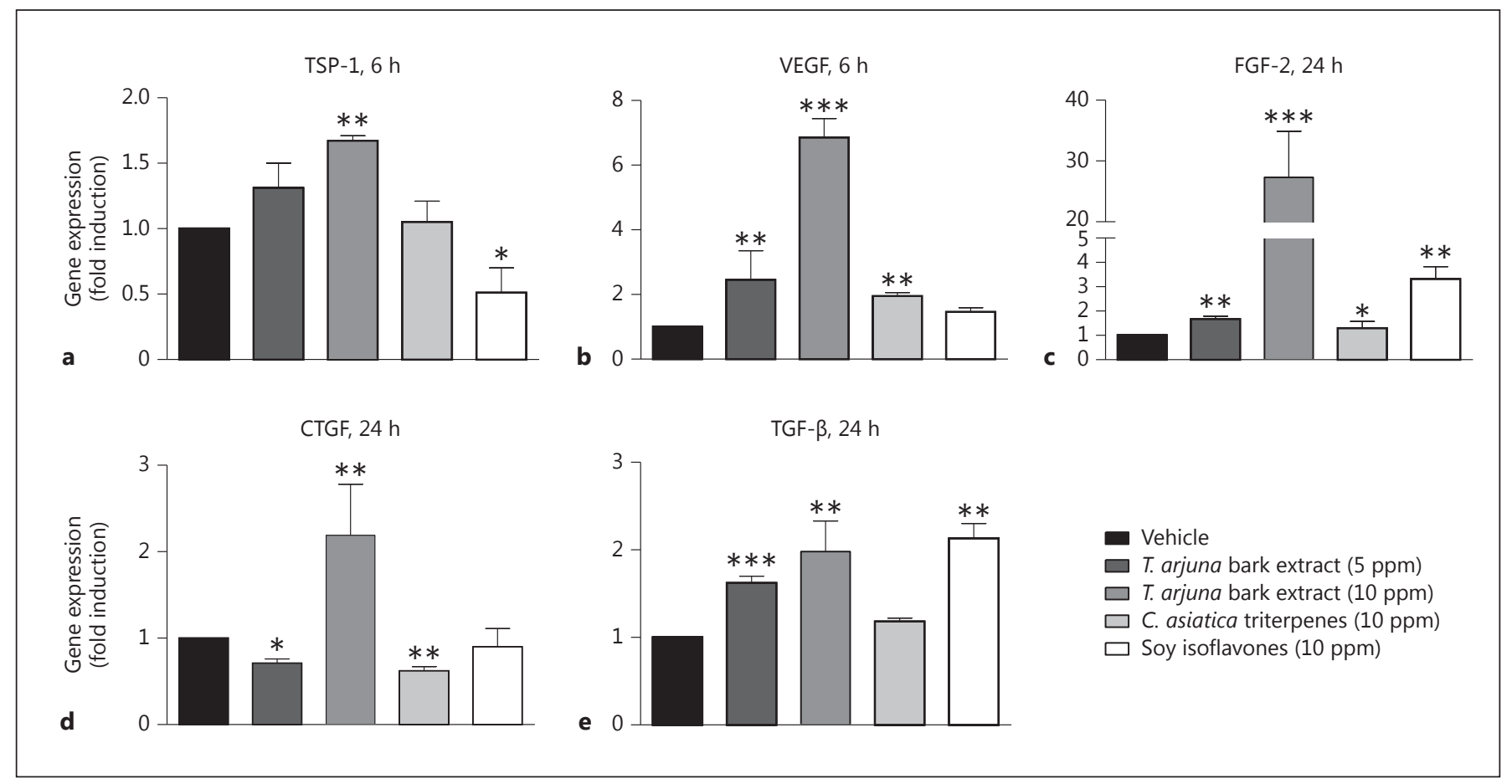

Fig. 2. TSP-1 (a), VEGF (b), FGF-2 (c), CTGF (d), TGF- $\beta$ (e) gene expression in human epidermal keratinocytes after treatment with test substances at different time points measured by qRT-PCR. The results are calculated relative to vehicle and normalized to GAPDH gene expression. ${ }^{*} \mathrm{p}<0.05,{ }^{* *} \mathrm{p}<0.01,{ }^{* * *} \mathrm{p}<0.001$ vs. vehicle.

Treatment of human epidermal keratinocytes with 10 ppm of T. arjuna bark extract significantly increased TSP1 and VEGF gene expression compared to vehicle after $6 \mathrm{~h}$ of application (fig. 2a, b). While incubation with C. asiati$c a$ triterpenes also led to significant increases in VEGF expression, administration of soy isoflavones did not significantly alter expression of this gene. Significant increases in FGF-2, CTGF and TGF- $\beta$ expression could be detected after $24 \mathrm{~h}$ of incubation with 5 and $10 \mathrm{ppm}$ of T. arjuna bark extract. Also, treatment with C. asiatica triterpenes was associated with significant increases in FGF-2 and CTGF at $24 \mathrm{~h}$ after administration. Incubation with soy isoflavones led to significant upregulation of FGF- 2 and TGF- $\beta$ and significant decrease in TSP-1 expression (fig. 2a, c-e).

Treatment of human epidermal keratinocytes with 5 ppm of T. arjuna bark extract further resulted in significant VEGF protein production compared to vehicle. Even though $10 \mathrm{ppm}$ of $T$. arjuna bark extract also increased VEGF levels, this increase was not statistically significant (fig. 3a).

Microarray analysis of human dermal fibroblasts treated with different concentrations of $T$. arjuna bark
Table 1. Excerpt of genes regulated in human dermal fibroblasts treated by test substances

\begin{tabular}{lll}
\hline & $\begin{array}{l}\text { Regulation } \\
\text { of MMP-1 }\end{array}$ & $\begin{array}{l}\text { Regulation } \\
\text { of IL-8 }\end{array}$ \\
\hline T. arjuna bark extract, 5 ppm & -1.73 & -2.30 \\
T. arjuna bark extract, $10 \mathrm{ppm}$ & -2.20 & -2.73 \\
C. asiatica triterpenes, $10 \mathrm{ppm}$ & -2.82 & -3.37 \\
Soy isoflavones, $10 \mathrm{ppm}$ & -3.04 & -6.04 \\
TGF- $\beta, 10 \mathrm{ng} / \mathrm{ml}$ & -3.05 & -3.54 \\
\hline
\end{tabular}

${ }^{\mathrm{a}} \mathrm{x}$-fold compared to vehicle.

extract revealed a dose-dependent, more than 2 -fold downregulation of IL-8 and MMP-1 gene expression compared to vehicle control (table 1). Both reference substances, C. asiatica triterpenes and soy isoflavones, also reduced the gene expression of these markers 3- to 6-fold. The positive control TGF- $\beta$ showed a 3 -fold reduction in IL- 8 and MMP-1 gene expression. 


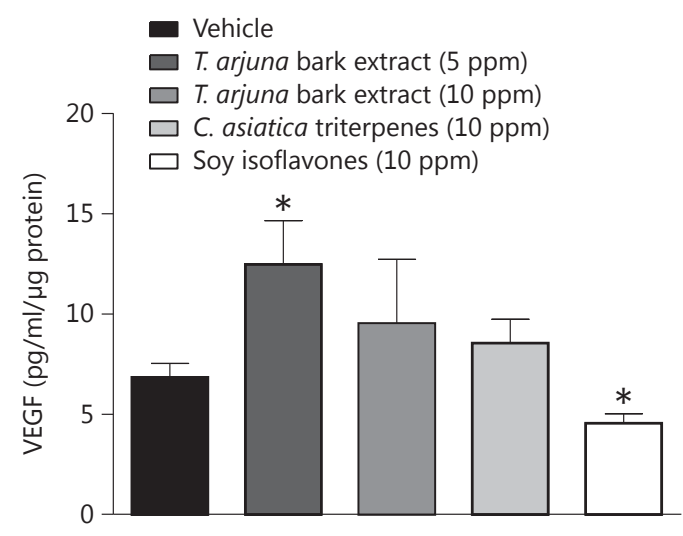

Fig. 3. Relative VEGF release in cell culture supernatant $24 \mathrm{~h}$ after application of test substances. ${ }^{*} \mathrm{p}<0.05$ vs. vehicle.
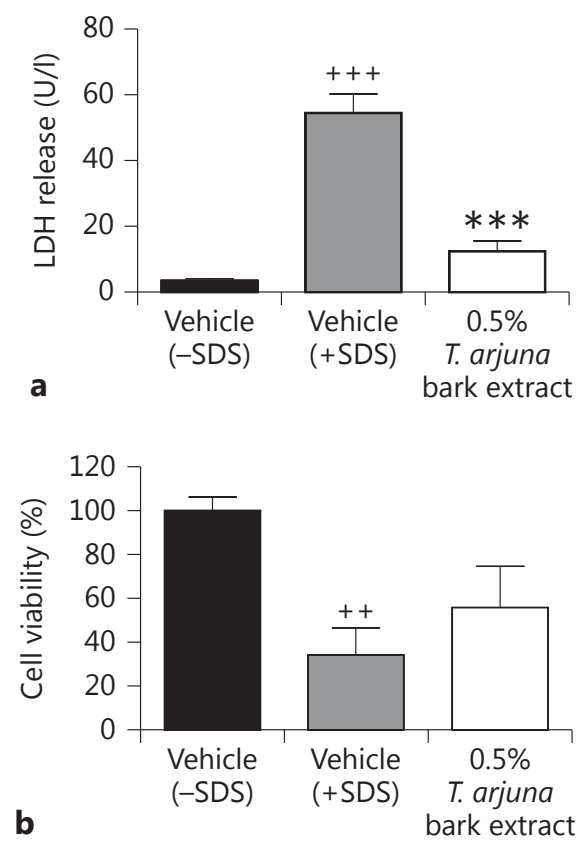

Fig. 4. LDH release and cell viability after SDS challenge. a LDH release $48 \mathrm{~h}$ after application of test substances. b Cell viability (XTT assay) $52 \mathrm{~h}$ after application of test substances. Student's t test: ${ }^{++} \mathrm{p}<0.01,{ }^{+++} \mathrm{p}<0.001$ vs. vehicle (-SDS), ${ }^{* * *} \mathrm{p}<0.001$ vs. vehicle $(+\operatorname{SDS})$.

\section{T. arjuna Bark Extract Protects Epidermal Cells against External Stress}

SDS challenge of reconstituted human epidermal skin resulted in a significantly increased $\mathrm{LDH}$ release after $48 \mathrm{~h}$, indicating membrane defects of the cells. Application of T. arjuna bark extract prior to SDS challenge markedly reduced LDH release by 78\% (fig. 4a). Also, incubation with SDS significantly reduced cell viability by approximately $66 \%$, which was strongly diminished by $T$. arjuna bark extract pretreatment (fig. $4 \mathrm{~b}$ ).

\section{Penetration Properties and Bioavailability of}

T. arjuna Bark Extract

Penetration of $T$. arjuna bark extract was very high (approx. 50\%) when the oil phase of the emulsion was based on a polar emollient like PPG-3 myristyl ether. The good bioavailability could be improved up to about $70 \%$ when $T$. arjuna bark extract was added to the formulation as a solution in 1,2-pentanediol (TEGO ${ }^{\circledR}$ Arjuna S). Formulating T. arjuna bark extract in the oil phase of an emulsion with a medium polar emollient (caprylic/capric triglyceride) led to a bioavailability of approximately $12 \%$. However, when $\mathrm{TEGO}^{\circledR}$ Arjuna $\mathrm{S}$ (solution of $T$. arjuna bark extract in 1,2-pentanediol) was used instead, an almost 4 -fold increased penetration was achieved. Similar results were obtained when the relatively non-polar mineral oil was employed in the emulsion. The bioavailability of $T$. arjuna bark extract was raised from approximately $14 \%$ to more than $25 \%$ when using the novel product form of TEGO ${ }^{\circledR}$ Arjuna $\mathrm{S}$ in this formulation (fig. 5a).

In addition, the formulation properties of $T$. arjuna bark extract can be drastically improved by employing the product as a solution in 1,2-pentanediol $\left(\mathrm{TEGO}^{\circledR}\right.$ Arjuna $\mathrm{S})$. Figure 5 shows microscopic pictures of an $\mathrm{O} / \mathrm{W}$ emulsion where the pure T. arjuna bark extract was dissolved in the hot oil phase of the emulsion (fig. 5b) in comparison to adding TEGO ${ }^{\circledR}$ Arjuna $S$ to the emulsion at the end of the production process (fig. $5 \mathrm{c}$ ). It can easily be seen that the use of T. arjuna bark extract without 1,2 pentanediol in the oil phase led to formation of crystals in the finished formulation. By contrast, no crystallization was observed when TEGO ${ }^{\circledR}$ Arjuna $S$ was employed.

\section{Administration of T. arjuna Bark Extract Improves \\ Skin Moisturization and Elasticity in vivo}

In vivo data revealed that administration of T. arjuna bark extract for 4 weeks led to a marked decrease in TEWL by approximately $46 \%$ compared to vehicle (fig. 6a). Also, daily treatment with $T$. arjuna bark extract for 4 weeks 
Fig. 5. (a) Penetration profile of T. ariuna bark extract in comparison to TEGO ${ }^{\circledR} \mathrm{Ar}$ juna S. Shown are microscopic images of formulations prepared by (b) disolving pure T. Arjuna bark extracts or (c) the solution in pentanediol $\left(\mathrm{TEGO}^{\circledR}\right.$ Arjuna $\mathrm{S}$ ) out of a formulation with different oil phases. Skin penetration is calculated as percent bioavailability in porcine skin.

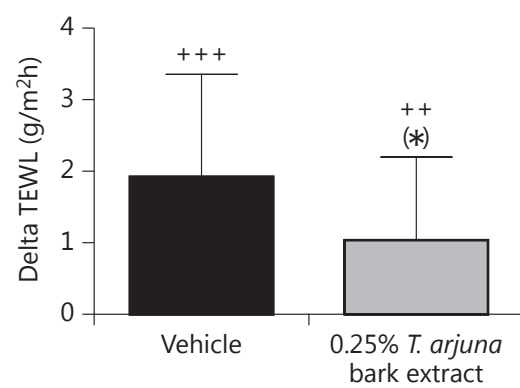

a

Fig. 6. TEWL (a), skin moisturization (b) and skin scaliness (c) after 4 weeks of application of $T$. arjuna bark extract. Student's t test: ${ }^{+++} \mathrm{p}<0.001{ }^{++} \mathrm{p}<0.01$ vs. start, ${ }^{+} \mathrm{p}<0,05,(+) \mathrm{p}<0.1$ vs. start, ${ }^{*} \mathrm{p}<$ $\left.0.05,{ }^{*}\right) \mathrm{p}<0.1$ vs. vehicle $(\mathrm{n}=30)$. Exemplary image of the inner forearm of a volunteer taken by Visioscan before application (d) and 4 weeks after application (e) of the test formulation with $0.25 \% \mathrm{~T}$. arjuna bark extract.

Pentacyclic Triterpenes from T. arjuna Show Benefits on Aged and Dry Skin
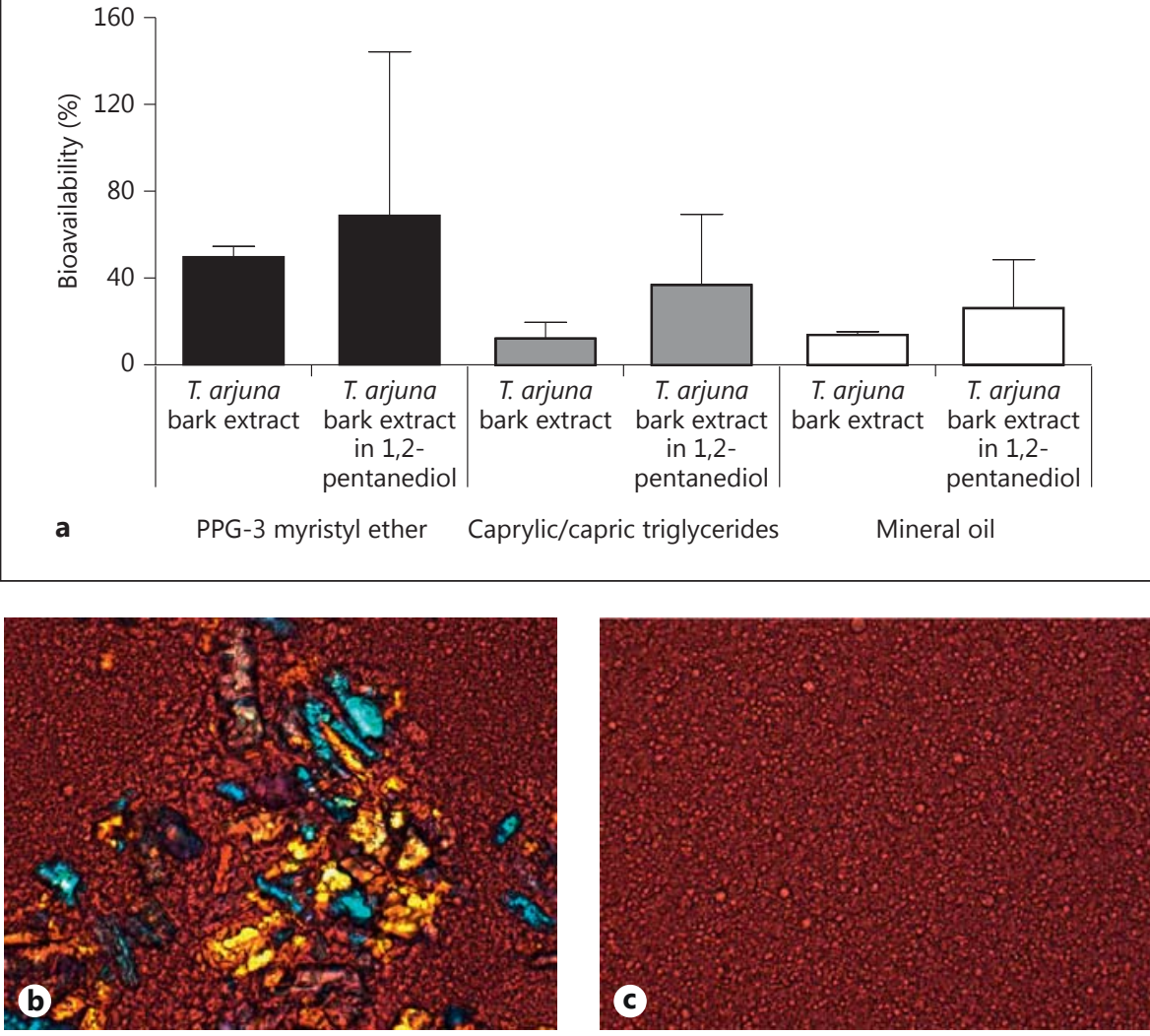

a

PPG-3 myristyl ether Caprylic/capric triglycerides Mineral oil
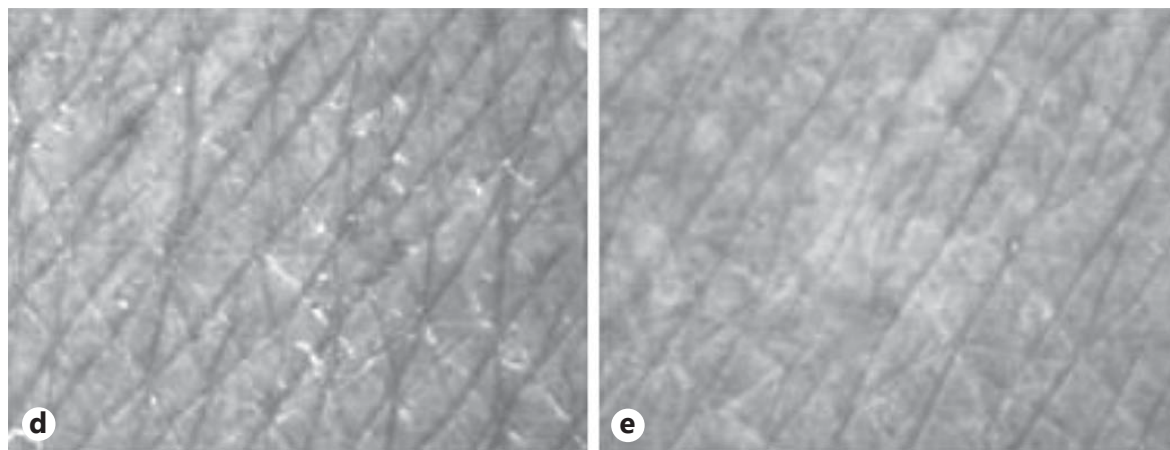

Skin Pharmacol Physiol 2014;27:71-81 DOI: $10.1159 / 000351387$ 
Fig. 7. Skin elasticity after 8 weeks of application of T. arjuna bark extract. a Elasticity parameter R1. b Elasticity parameter R5. Student's t test: ${ }^{+} \mathrm{p}<0.05$ vs. start $(\mathrm{n}=30)$.
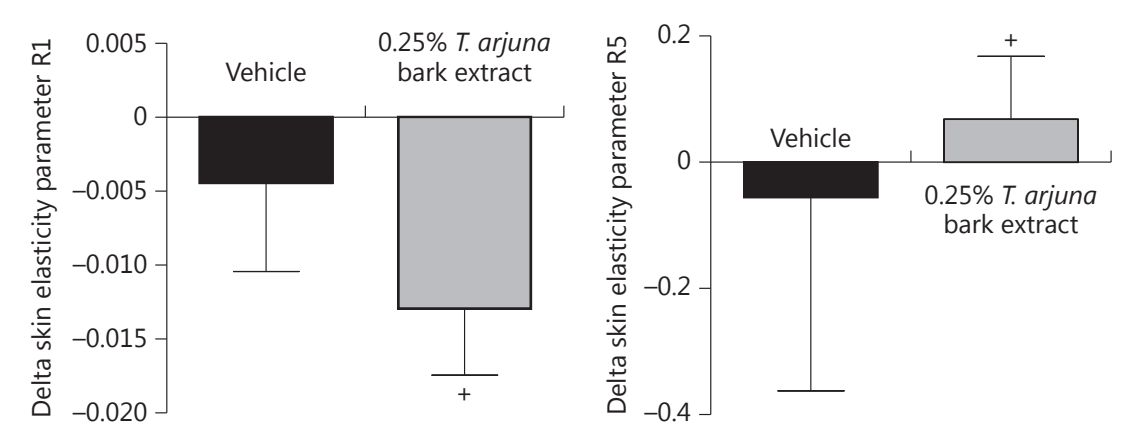

a

b
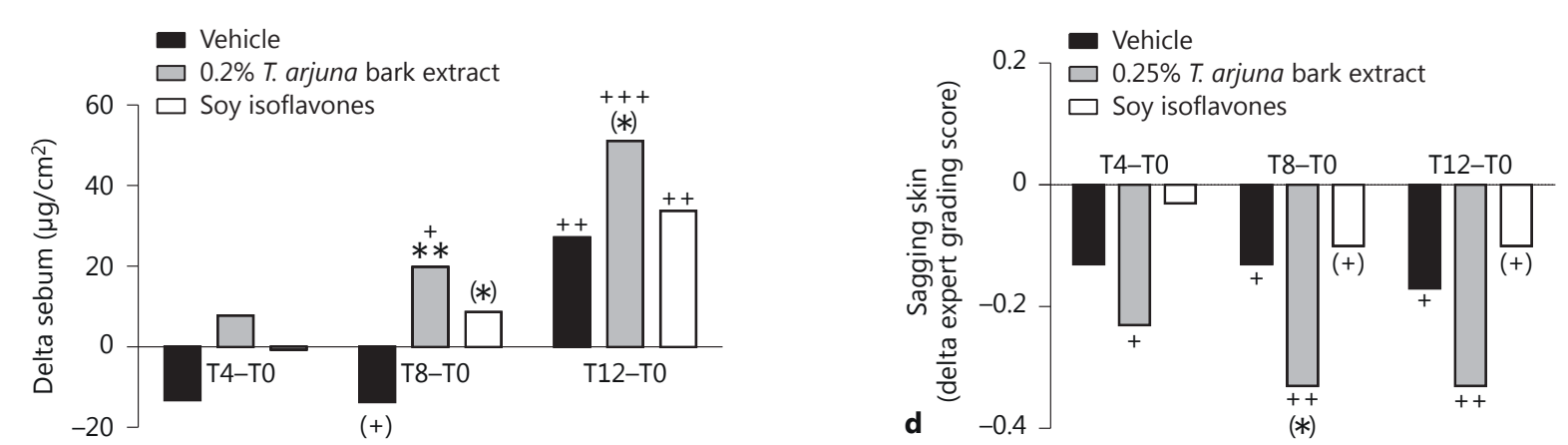

a
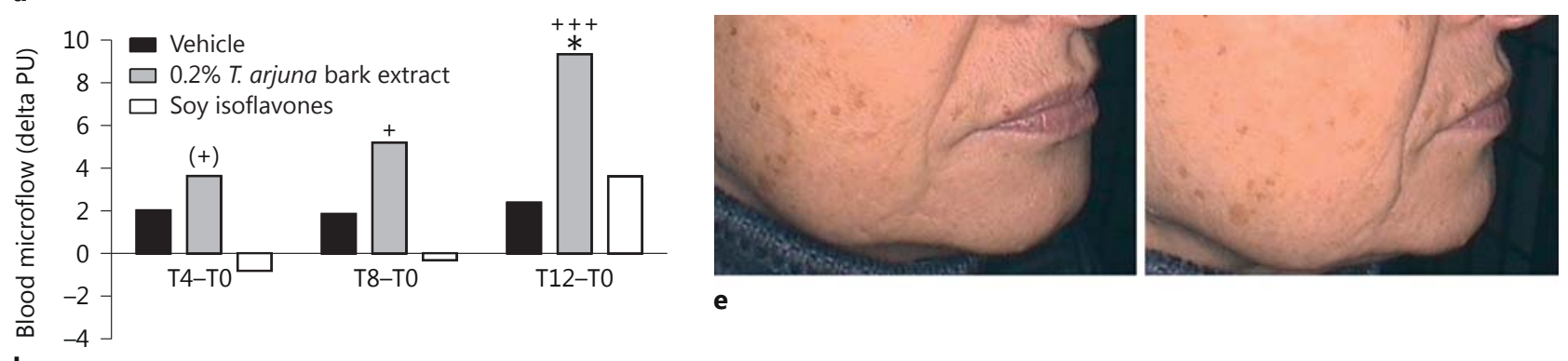

b
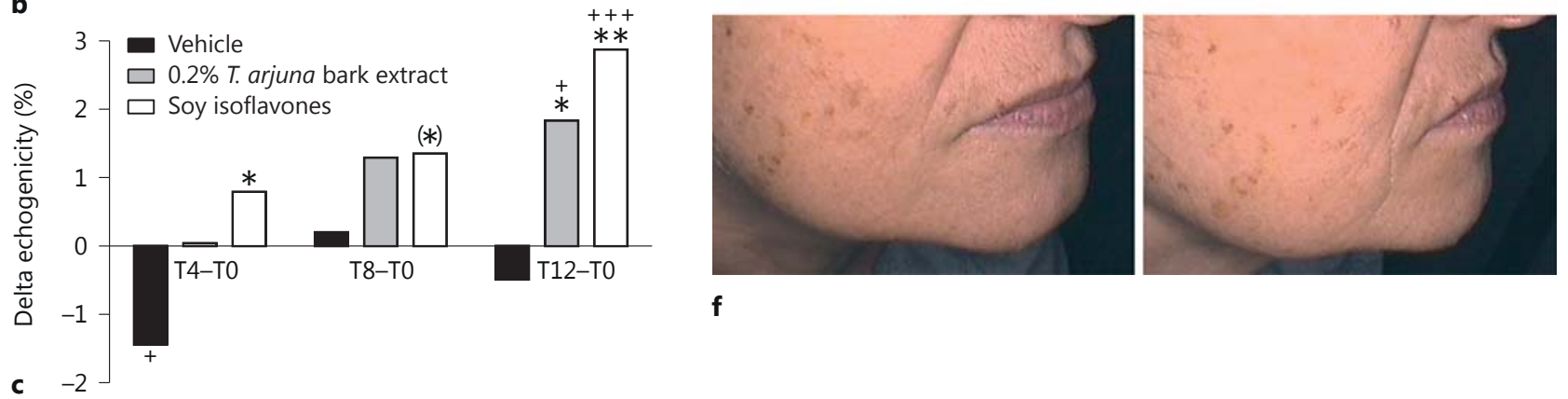

Fig. 8. Amount of sebum on the skin (a), skin blood microflow (b), skin density/echogenicity (c) and decrease of sagging skin (d) relative to the beginning after 4,8 , and 12 weeks of application of $T$. arjuna bark extract. PU $=$ Perfusion units. Student's t test: ${ }^{+++} \mathrm{p}<$
$0.001,{ }^{++} \mathrm{p}<0.01,{ }^{+} \mathrm{p}<0.05,{ }^{(+)} \mathrm{p}<0.1$ vs. start; $\left.{ }^{* *} \mathrm{p}<0.01,{ }^{*}\right) \mathrm{p}<$ 0.1 vs. vehicle $(n=30)$. Digital images of a representative volunteer before (e) and after 12 weeks (f) of application of T. arjuna bark extract. 
was associated with a significant 4 -fold increase in corneometer units compared to vehicle, indicating improved skin moisturization (fig. 6b). Scaliness was reduced by administration of both vehicle and $0.25 \%$ T. arjuna bark extract. However, the latter led to more pronounced reduction, which was also visualized by digital images (fig. 6c-e).

Daily application of $0.25 \%$ T. arjuna bark extract for 8 weeks was also associated with significantly improved skin elasticity as indicated by a significant reduction of R1 and a significant increase of R5 (fig. 7a, b).

\section{Administration of T. arjuna Bark Extract Reduces}

Symptoms of Postmenopausal Skin in vivo

In the subsequent in vivo target study including exclusively postmenopausal women, administration of $T$. arjuna bark extract for a period of 8 and 12 weeks increased facial skin sebum content by 24 and $61 \%$, respectively, compared to baseline (fig. 8a). Although application of soy isoflavones also evoked an enhanced sebum content, this increase was not statistically significant compared to vehicle. Similar results were obtained with respect to skin blood microcirculation: while daily application of T. arjuna bark extract led to a significantly increased blood microflow, administration of soy isoflavones did not show significant alterations of blood microcirculation compared to vehicle (fig. 8b). In vivo data also demonstrated significantly improved skin thickness/echogenicity after 12 weeks of application of T. arjuna bark extract. However, similar results were obtained with soy isoflavones (fig. 8c). Visual and tactile clinical evaluation of skin sagging by a dermatologist confirmed the results of the objective measurements and revealed a significant reduction of skin sagging after 4, 8 and 12 weeks of daily application of T. arjuna bark extract. Neither the administration of soy isoflavones nor vehicle could significantly alter skin sagging (fig. 8d). The visible reduction of sagging skin on the jowls by T. arjuna bark extract was also demonstrated using digital photography (fig. 8e, f).

\section{Discussion}

Based on the various known beneficial effects of pentacyclic triterpenoids on the skin [12-14], we aimed to determine their effects on cutaneous aging using various objective in vitro and in vivo measurements.

Cutaneous aging may be considered as the accumulation of different deleterious changes in cells and tissues [1] and occurs in all individuals at a variable rate that is

Pentacyclic Triterpenes from T. arjuna

Show Benefits on Aged and Dry Skin influenced by genetic, environmental and hormonal factors and may thus be divided into two basic processes: intrinsic aging, also referred to as chronological aging, and extrinsic aging. Extrinsic aging primarily arises from chronic UV light exposure, while, for example, hormonal changes during perimenopausal years clearly contribute to intrinsic aging [20]. Nevertheless, both types of cutaneous aging share several molecular similarities including formation of reactive oxygen species and the induction of MMPs [1]. Reactive oxygen species may induce expression of MMP-1, -3 and -9 via activation of c-Jun and activator protein-1 and reduce the expression of procollagen-1 [21]. Indeed, increased levels of partially degraded collagen have been found in intrinsically aged and photoaged skin. UV radiation also activates nuclear factor kappa B that stimulates the transcription of proinflammatory cytokine genes such as IL-1b, TNF-a, IL-6 and IL-8 [22].

Importantly, treatment with $T$. arjuna bark extract led to altered gene expression in cutaneous cells in vitro. Specifically, incubation of human dermal fibroblasts with $T$. arjuna bark extract resulted in a dose-dependent, more than 2-fold downregulation of MMP-1 and IL-8 gene expression, indicating inhibitory effects on MMP-1 and proinflammatory cytokine activities. Also, treatment of human epidermal keratinocytes with T. arjuna bark extract resulted in significant increases in VEGF, TSP-1, FGF-2, CTGF and TGF- $\beta$ mRNA expression as well as enhanced VEGF protein production. Notably, changes in mRNA expression were more pronounced upon $T$. arjuna bark extract incubation compared to incubation with C. asiatica triterpenes or soy isoflavones. Similar increases in TSP-1, VEGF and FGFR2 gene expression were noted after treatment of reconstituted human epidermis with T. arjuna bark extract. Thus, alteration of these genes may represent some potential mechanisms of action of T. arjuna bark extract in the prevention of cutaneous aging, since it was recently demonstrated that reduction of CTGF and impaired TGF- $\beta$ /Smad signalling may significantly contribute to diminished type-I procollagen in intrinsically aged skin [23]. FGF-2 induces endothelial cell proliferation and migration. Moreover, it increases the synthesis of matrix macromolecules and hyaluronic acid by stimulating the gene expression of hyaluronan synthases [24]. FGF-2 also contributes to the woundhealing process by stimulating fibroblast proliferation and by inhibiting the expression of MMP-1 in keratinocytes [25]. FGF-2 and its receptor FGFR2 may be also related to enhanced sebaceous gland activity with increased sebum production [26]. Its upregulation in keratinocytes upon incubation with T. arjuna bark extract may thus 
contribute to the increased skin sebum content as observed in our clinical experiments.

VEGF represents a potent angiogenic factor [27] and its 121-, 165- and 189-amino-acid isoforms are predominantly found in human keratinocytes [28]. VEGF promotes endothelial proliferation during formation of new blood vessels. Physiologically, VEGF is expressed at relatively low levels in adult skin. Insufficient oxygen supply in the skin induces upregulation of VEGF gene expression by epidermal keratinocytes and dermal fibroblasts. Postmenopausal and photoaged skin have been reported to display an age-dependent reduction of cutaneous microvasculature, which may explain several of the physiological alterations that are characteristic of old skin, including pallor, decreased skin temperature, reduced cutaneous vascular responsiveness and decreased nutritional supply $[29,30]$. Thus, a moderate induction of VEGF favouring a better blood supply - as shown by administration of T. arjuna bark extract in vitro and in vivo - may be warranted.

TSP-1 is a large multimeric ECM protein and has been well characterized as a potent endogenous regulator in epithelial tissues, including the skin [31]. Its epidermal overexpression has been recently shown to inhibit dermal photodamage, as well as collagen and elastic fibre disorganization, thus potentially preventing the formation of skin wrinkles [32].

As demonstrated in our experiments, $T$. arjuna bark extract was easily incorporated into cosmetic emulsions when applied as a solution in pentanediol $\left(\mathrm{TEGO}^{\circledR} \mathrm{Ar}-\right.$ juna S). An additional benefit of this product form is the enhanced bioavailability, which was also increased when formulating with more polar emollients [33].

The suggested effects of $T$. arjuna bark extract demonstrated in vitro were then further evaluated in vivo with respect to potential antiaging properties using a representative patient population. Instrumental and clinical evaluations of patients included into the clinical study showed significant improvements in skin moisturization and elasticity as well as a reduction in scaliness with results better than those of the vehicle group. Sensitive, aged and dry skin is associated with increased TEWL, increased penetrability and higher susceptibility to irritants, leading to impaired barrier function. In vivo data revealed that administration of $T$. arjuna bark extract led to a marked decrease in TEWL compared to vehicle, indicating recovery of epidermal barrier function.

We also could demonstrate a strong beneficial effect of T. arjuna bark extract on postmenopausal skin. Administration of T. arjuna bark extract significantly increased skin sebum content and skin blood microcirculation compared to vehicle. Although application of soy isoflavones also evoked an enhanced sebum content, this increase was not statistically significant compared to vehicle. In vivo data also demonstrated significantly improved skin thickness/echogenicity after 12 weeks of application of T. arjuna bark extract similar to that of patients receiving soy isoflavones. Moreover, daily administration of $T$. arjuna bark extract revealed a significant reduction of skin sagging, which was not observed upon administration of soy isoflavones or vehicle.

Soy isoflavones are known to have various biological effects through oestrogen receptor activity and are often used in cosmetic formulation for mature skin [34, 35]. However, they contain estrogenic metabolites and possess phytohormonal activity, which is often not desired. Also, their precise mechanism of action in skin is still unknown, and their possible side effects have not been well investigated [3]. T. arjuna bark extract has a comparable activity on cutaneous ageing without influencing hormone metabolism, as demonstrated in an oestrogen receptor-binding study (data not shown).

\section{Conclusion}

T. arjuna bark extract appears as an innovative active ingredient that alters various genes in vitro which are involved in cutaneous ageing and has been demonstrated to exert various antiaging properties in vivo.

\section{Disclosure Statement}

This study was funded by Evonik Industries AG, Essen, Germany.

\footnotetext{
References

1 Kohl E, Steinbauer J, Landthaler M, Szeimies RM: Skin ageing. J Eur Acad Dermatol Venereol 2011;25:873-884.

2 Makrantonaki E, Zouboulis CC: William J Cunliffe Scientific Awards. Characteristics and pathomechanisms of endogenously aged skin. Dermatology 2007;214:352-360.

3 Verdier-Sevrain S: Effect of estrogens on skin aging and the potential role of selective estrogen receptor modulators. Climacteric 2007; 10:289-297.

4 Uitto J: Understanding premature skin aging. New Engl J Med 1997;337:1463-1465.
}

Farwick et al. 
5 Quatresooz P, Pierard GE: Immunohistochemical clues at aging of the skin microvascular unit. J Cutan Pathol 2009;36:39-43.

-6 Lavker RM: Structural alterations in exposed and unexposed aged skin. J Invest Dermatol 1979;73:59-66.

7 Pavicic T, Gauglitz GG, Lersch P, SchwachAbdellaoui K, Malle B, Korting HC, Farwick M: Efficacy of cream-based novel formulations of hyaluronic acid of different molecular weights in anti-wrinkle treatment. J Drugs Dermatol 2011;10:990-1000.

$>8$ Goldberg RA, McCann JD, Fiaschetti D, Ben Simon GJ: What causes eyelid bags? Analysis of 114 consecutive patients. Plast Reconstr Surg 2005;115:1395-1402, discussion 14031394.

$>9$ James JT, Dubery IA: Pentacyclic triterpenoids from the medicinal herb, Centella asiatica (L.) Urban. Molecules 2009; 14:39223941.

10 Liu J, Liu Y, Parkinson A, Klaassen CD: Effect of oleanolic acid on hepatic toxicant-activating and detoxifying systems in mice. J Pharmacol Exp Ther 1995;275:768-774.

$\checkmark 11$ Huang MT, Ho CT, Wang ZY, Ferraro T, Lou YR, Stauber K, Ma W, Georgiadis C, Laskin JD, Conney AH: Inhibition of skin tumorigenesis by rosemary and its constituents carnosol and ursolic acid. Cancer Res 1994;54: 701-708.

$>12$ Lee HK, Nam GW, Kim SH, Lee SH: Phytocomponents of triterpenoids, oleanolic acid and ursolic acid, regulated differently the processing of epidermal keratinocytes via PPAR-a pathway. Exp Dermatol 2006;15:6673.

$\checkmark 13$ Lim SW, Hong SP, Jeong SW, Kim B, Bak H, Ryoo HC, Lee SH, Ahn SK: Simultaneous effect of ursolic acid and oleanolic acid on epidermal permeability barrier function and epidermal keratinocyte differentiation via peroxisome proliferator-activated receptor- $\alpha$. J Dermatol 2007;34:625-634.

14 Rane MM, Mengi SA: Comparative effect of oral administration and topical application of alcoholic extract of Terminalia arjuna bark on incision and excision wounds in rats. Fitoterapia 2003;74:553-558.
15 Mehul B, Asselineau D, Bernard D, Leclaire J, Regnier M, Schmidt R, Bernerd F: Gene expression profiles of three different models of reconstructed human epidermis and classical cultures of keratinocytes using cDNA arrays. Arch Dermatol Res 2004;296:145-156.

16 Langmann T, Moehle C, Mauerer R, Scharl M, Liebisch G, Zahn A, Stremmel W, Schmitz G: Loss of detoxification in inflammatory bowel disease: dysregulation of pregnane $\mathrm{X}$ receptor target genes. Gastroenterology 2004; 127:26-40.

17 Langmann T, Mauerer R, Zahn A, Moehle C, Probst M, Stremmel W, Schmitz G: Real-time reverse transcription-PCR expression profiling of the complete human ATP-binding cas sette transporter superfamily in various tissues. Clin Chem 2003;49:230-238.

18 Jachowicz J, McMullen R, Prettypaul D: Alteration of skin mechanics by thin polymer films. Skin Res Technol 2008;14:312-319.

19 Akhtar N, Zaman SU, Khan BA, Amir MN, Ebrahimzadeh MA: Calendula extract: effects on mechanical parameters of human skin. Acta Pol Pharm 2011;68:693-701.

20 Verdier-Sevrain S, Bonte F, Gilchrest B: Biology of estrogens in skin: implications for skin aging. Exp Dermatol 2006;15:83-94.

21 Chung JH, Kang S, Varani J, Lin J, Fisher GJ, Voorhees JJ: Decreased extracellular signalregulated kinase and increased stress-activated MAP kinase activities in aged human skin in vivo. J Invest Dermatol 2000;115:177-182.

22 Senftleben U, Karin M: The IKK/NF- $\kappa$ B pathway. Crit Care Med 2002;30:S18-S26.

23 Quan T, Shao Y, He T, Voorhees JJ, Fisher GJ: Reduced expression of connective tissue growth factor (CTGF/CCN2) mediates collagen loss in chronologically aged human skin. J Invest Dermatol 2010;130:415-424.

24 Kuroda K, Utani A, Hamasaki Y, Shinkai H: Up-regulation of putative hyaluronan synthase mRNA by basic fibroblast growth factor and insulin-like growth factor-1 in human skin fibroblasts. J Dermatol Sci 2001;26:156160.

25 Pilcher BK, Gaither-Ganim J, Parks WC, Welgus HG: Cell type-specific inhibition of keratinocyte collagenase- 1 expression by basic fibroblast growth factor and keratinocyte growth factor. A common receptor pathway. J Biol Chem 1997;272:18147-18154.
6 Melnik BC, Schmitz G, Zouboulis CC: Antiacne agents attenuate FGFR2 signal transduction in acne. J Invest Dermatol 2009;129: 1868-1877.

-27 Yano K, Kajiya K, Ishiwata M, Hong YK, Miyakawa T, Detmar M: Ultraviolet B-induced skin angiogenesis is associated with a switch in the balance of vascular endothelial growth factor and thrombospondin-1 expression. J Invest Dermatol 2004;122:201-208.

28 Ballaun C, Weninger W, Uthman A, Weich $H$, Tschachler E: Human keratinocytes express the three major splice forms of vascular endothelial growth factor. J Invest Dermatol 1995;104:7-10.

29 Chung JH, Yano K, Lee MK, Youn CS, Seo JY, Kim KH, Cho KH, Eun HC, Detmar M: Differential effects of photoaging versus intrinsic aging on the vascularization of human skin. Arch Dermatol 2002;138:1437-1442.

30 Kelly RI, Pearse R, Bull RH, Leveque JL, de Rigal J, Mortimer PS: The effects of aging on the cutaneous microvasculature. J Am Acad Dermatol 1995;33:749-756.

31 Armstrong LC, Bornstein P: Thrombospondins 1 and 2 function as inhibitors of angiogenesis. Matrix Biol 2003;22:63-71.

-32 Yano K, Oura H, Detmar M: Targeted overexpression of the angiogenesis inhibitor thrombospondin-1 in the epidermis of transgenic mice prevents ultraviolet-B-induced angiogenesis and cutaneous photo-damage. J Invest Dermatol 2002;118:800-805.

33 Schiemann Y, Wegmann M, Lersch P, Heisler E, Farwick M: Polar emollients in cosmetic formulations enhance the penetration and biological effects of phytosphingosine on skin. Colloids Surf A Physicochem Eng Asp 2008; 331:103-107.

34 Jackson RL, Greiwe JS, Schwen RJ: Ageing skin: oestrogen receptor- $\beta$ agonists offer an approach to change the outcome. Exp Dermatol 2011;20:879-882.

35 Patriarca MT, Goldman KZ, Dos Santos JM, Petri V, Simoes RS, Soares JM Jr, Simoes MJ, Baracat EC: Effects of topical estradiol on the facial skin collagen of postmenopausal women under oral hormone therapy: a pilot study. Eur J Obstet Gynecol Reprod Biol 2007;130: 202-205.
Pentacyclic Triterpenes from T. arjuna Show Benefits on Aged and Dry Skin
Skin Pharmacol Physiol 2014;27:71-81 DOI: $10.1159 / 000351387$ 